

\section{Clostridium difficile infection}

Risk factors and potential vaccines

Antibiotics treatment is one of the main factors of risk for developing Clostridium difficile (C. diff) infection, a condition responsible for nearly 500,000 infections and 30,000 death

each year in the U.S. alone.

Sharma and their collaborators

at the Biotechnology Core

Laboratory of the Nation

Institute of Diabetes and

Digestive and Kidney Diseases

(NIDDK) optimised the

bioprocessing conditions for

overexpression of potentil

vaccine candidate rARU

fragment by expressing it in

E. coli at restricted dissolved

oxygen concentrations.

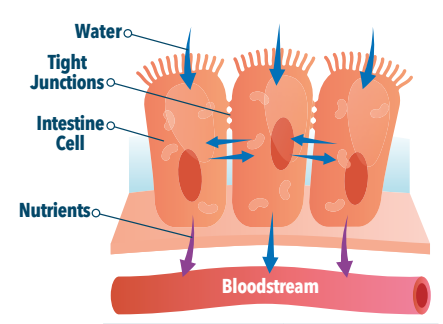

lostridium difficile (C. diff) has
become a common pathogen become a common pathogen that is responsible for healhcal causing life-threatening diarrhoea and coltis - an inflammation of the colon. for nearly 500,000 infections and 30,000 deaths each year. According to the U.S. Centres for Disease Control and Prevention, patients are 7 to 10 times more likely to get $C$. diff while on antibiotics or soon after. Treatment with antibiotics is commonly accompanied by gastrointestinal complications, particularly among the elderly. The duration of antibiotic treatment is one of the main factors of risk for developing $C$. diff infection. That is because antibiotics that fight bacterial infections by kiling pathogens can also heneficial bacteria in the gut

fora. Antibiotic-resistant C. diff, facing little to no competition from the gut flora, can then thrive as a result and muliply uncontrollably, causing the

Traditional treatments for $C$. diff infection include the use of metronidazole or vancomycin, strong antibiotics that are mited by their broad spectrum and further perturbation of the intestinal fora, which results in an unacceptab high recurrence rate. Emerging immunological therapies, including monoclonal antibodies and vaccines infection. Currently there is no FDA approved vaccine, but two potentia vaccine candidates from two major pharmaceutical companies are in clinical trials Phase ll. The production
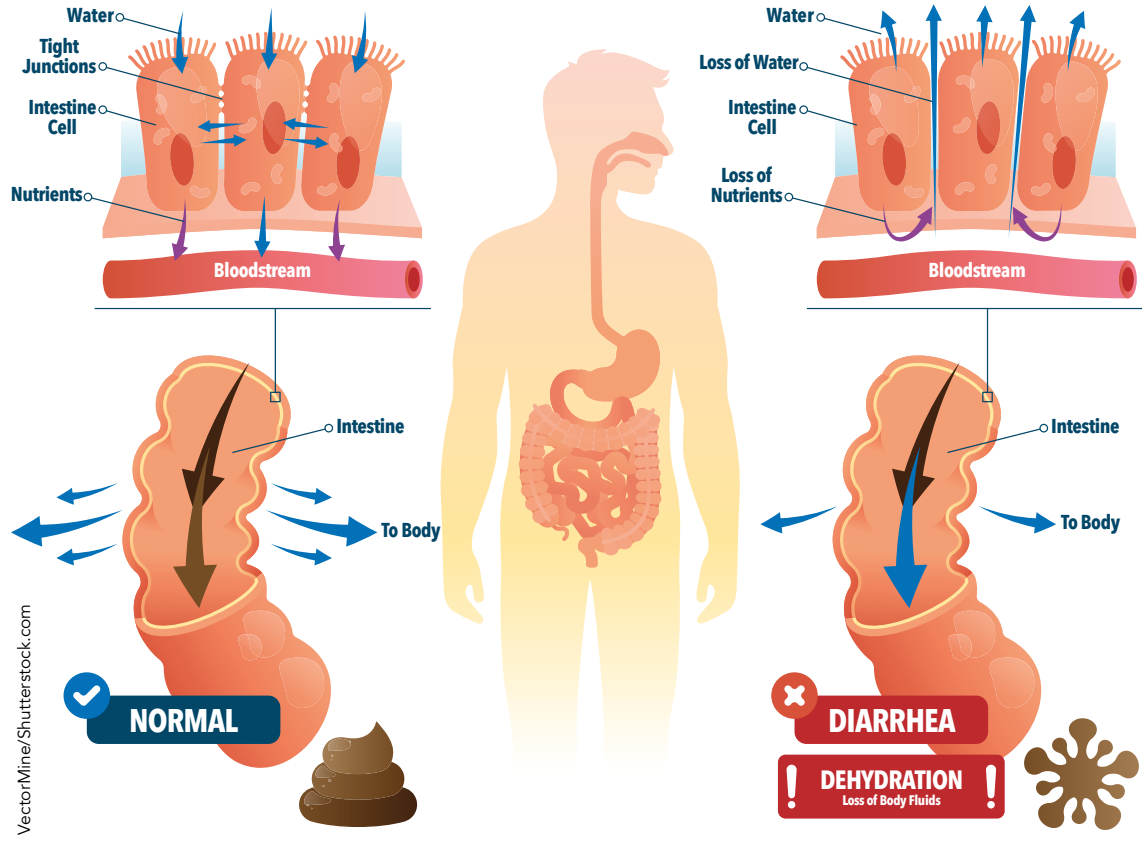

C. diff is a pathogen that can cause ilfe treatening dianthe and colitis. Antibiotic

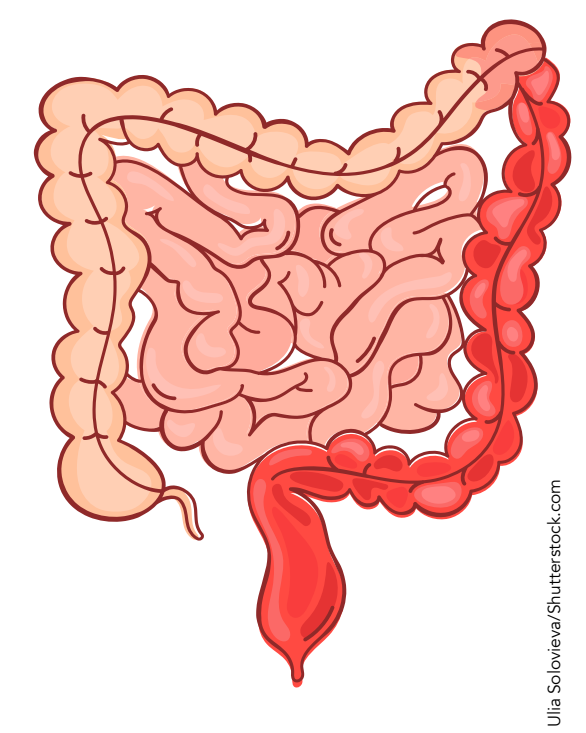

Ulcerative colitis is a long-term condition
that causes inflammation and ulcers in the
digestive tract.
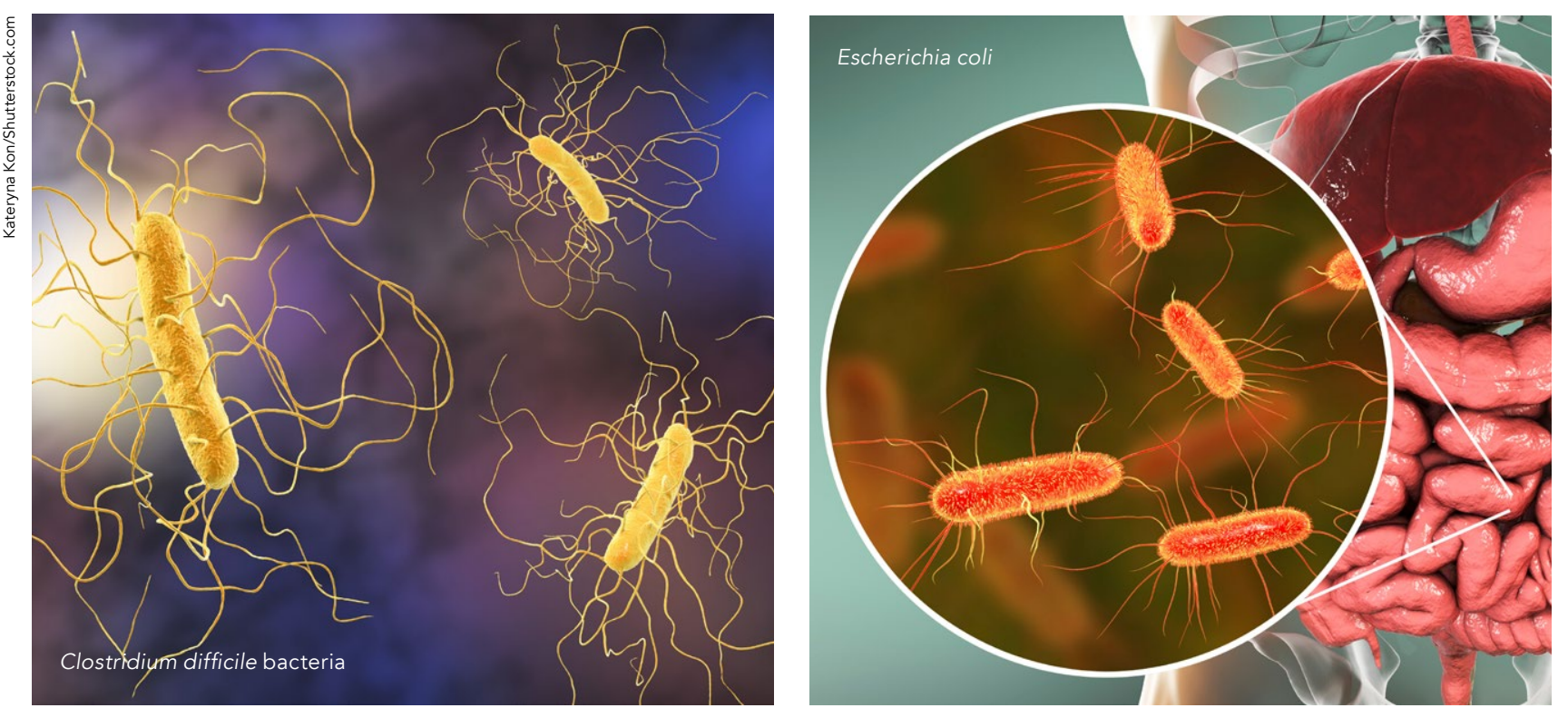

is responsible for the intestinal damage of the disease. The toxins, termed $A$

copied in high numbers and overexpressed within a host cell. One of production of recombinant proteins toxins. These potential vaccines con prokaryote Escherichia coli (E. coln.

ported in a paper published in 2020 E. colican be substantially increased in the presence of a restricted supply of dissolved oxygen (DO). When these conditions were applied the expression levels of rARU were up to 27-fold

region of $\mathrm{C}$. difft toxin $\mathrm{A}$, known as

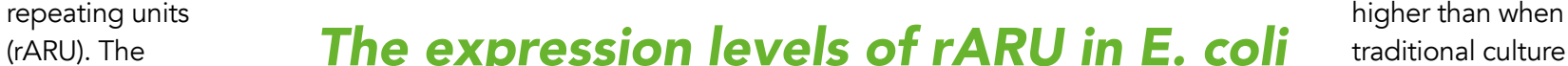
potential of rARU can be substantially increased in the growth conditions
as a candidate were implemented vaccine was shown presence of a restricted supply of In their study,

where antibodies dissolved oxygen. Sharma and their

showed immunity to $C$. diff infection and Clostridial proteins produced in $E$. neutralised the enterotoxic and cytotoxic coli is rather poor under standard activity of the toxin. bioprocess conditions. phe possen is ton for this special phen transcription at restricted DO, causing an increase in the exped

Dr Shiloach and Dr Sharma's team has (n) Birma and their, collaborators at the the National Institute of Diabetes and Digestive and Kidney Diseases (NIDDK) a branch of the National Institutes of Health (NIH), USA, specialise in pilot-scale production of biological compounds from various sources to advance research in fields such as vaccine and drug development. Drawing on their expertise, the research team developed a method that significantly mproved the difficult raRU expression.

\section{IMPROVING THE}

\section{BIOPROCESSING OF}

CLOSTRIDIAL VACCINE TARGETS

As is being done wh o

prons, ache,

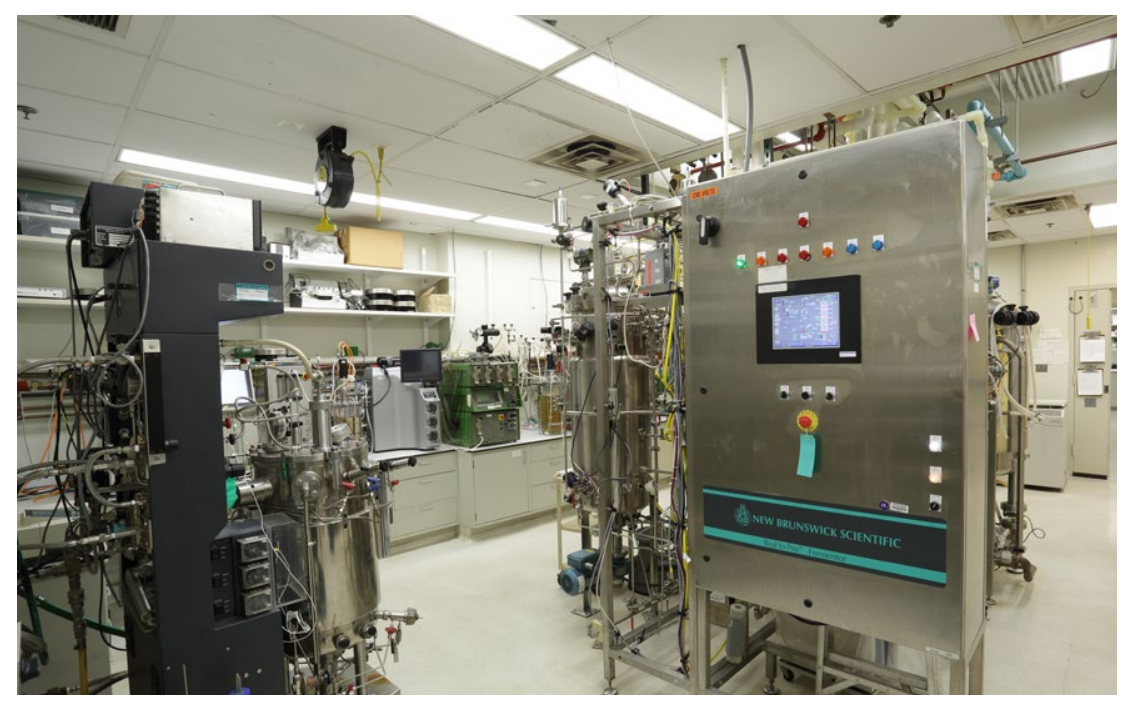

The researchers optimised the bioprocessing conditions for overexpression of potential vaccine 
ANKYRIN-LIKE PROTEINS

ARU HOST INFLUENC

Dr Shiloach, Dr Sharma and their

team showed that the cell wall

binding motifs in the rARU protein

sequence could potentially interact

with the host cellular proteins.

Among these proteins are some that act as repressors of transcription. particular, the interaction of rARU

with a transcriptional repressor known as ulaR can influence the biosynthesis of NADH and pentose sugars by affecting the L-ascorbate uptake.

The study also showed that some cellular peptides in the microbial host act as 'ankyrin-like proteins', which could potentially bind to the rARU protein. The ankyrin repeat is a The motif consists of 33 aminates. acids and the name ankyrin, which originates from the Greek word for 'anchor' gives away the structural significance of this important amino acid sequence. Ankyrincontaining proteins were discovered in mammalian cells and it became apparent that they were involved in cell signalling and regulation, and in stabilising the structural integrity of the plasma membrane. The authors found that the expression of the E. coli ankyrin proteins (yahD and ArpA) was higher at restricted dissolved oxygen than at unrestricted dissolved oxygen, and that the higher higher rARU expression (27 fotd). The results determine that the higher TARU expression is linked to interaction of the cell wall binding motifs in rARU with the ankyrin repeat containing proteins; however, the specific mechanism needs to be investigated.

\section{DOWNREGULATION OF KEY} UNRESTRICTED DISSOLVED OXYGE

An important finding of the team was that the enzyme RNA polymerase and key metabolites are downregulated in the presence of unrestricted oxygen. The details of the downregulated pathways can be seen in Figure 1 . The availability of ribosomes, amino acids, an moacyl-RNA, and ATP
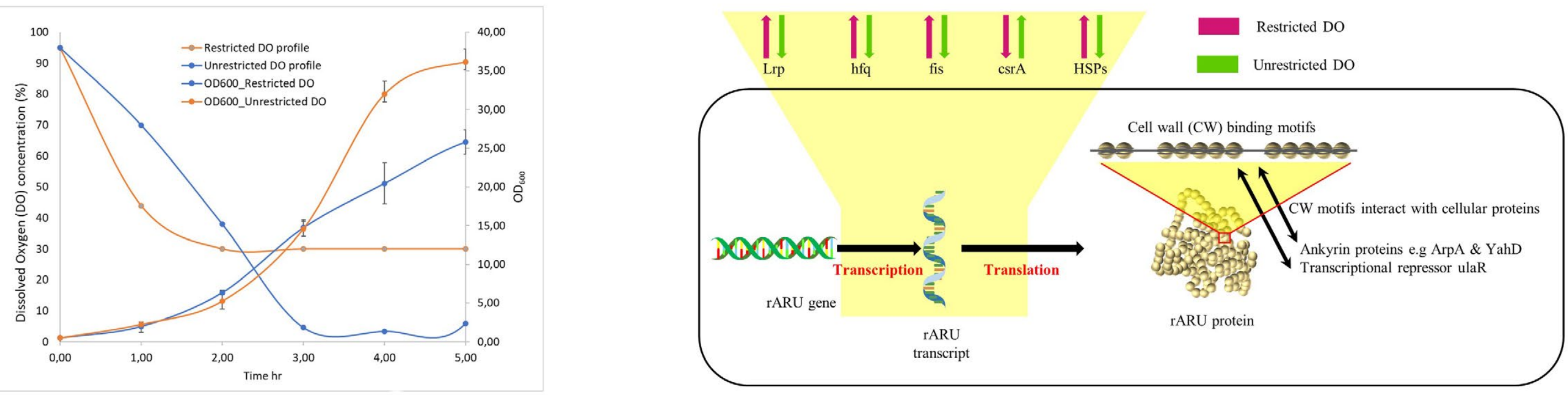

Growth and dissolved oxygen profiles

Schematic showing chandes in the major regulators and possible interaction of rARU with cellular proteins
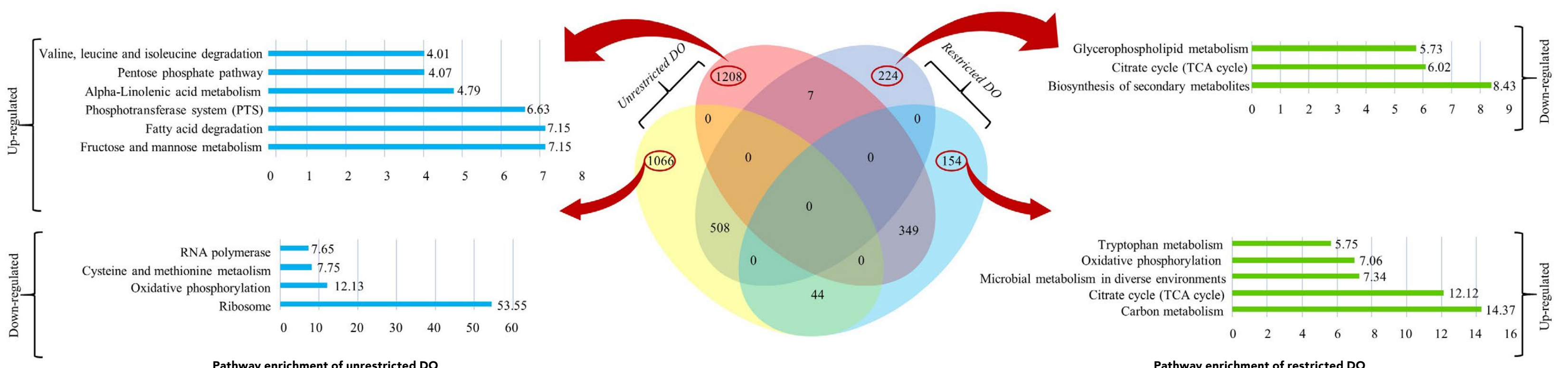

Pathway enrichment of unrestricted DO

Figure 1. Pathway analysis and interaction of rARU cell wall binding motifs with cellular proteins.

Pathway enrichment of restricted DO
The enzyme RNA polymerase and key metabolites in the host are downregulated in the presence of unrestricted oxygen.

in the unrestricted DO. The downregulation of genes associated with energy-related metabolism, central metabolism and ribosomal RNA is known to be associated with the cellular stress response generated overexpression
An additional transcriptional regulator that may have a possible role at chaperone molecule known as hfa. This molecule binds to small RNA sequences and is involved in the stabilisation of mRNA. In the study,
hfa was up-regulated 1.34 -fold at different DO conditions is the RNA restricted DO, thereby potentially expression

\section{CONCLUDING REMARKS}

The team of researchers led by $\mathrm{Dr}$ Shiloach and Dr Sharma optimised the bioprocessing conditions for overexpression of potential vaccine candidate TARU against infection of Clostridium difficile, for life-threatening diarrhoea and pseudomembranous colitis.

Recombinant rARU, a partial fragment of Toxin $A$ from the anaerobic enhancing the levels of rARU a bacterial pathogen responsible bacterium C. diff was expressed bacteria were grown at restricted DO concentrations. Therefore, an optimised expression was established by supplying the growing culture with an aeration system that generated restricted DO conditions.

While large bioreactors consume vast amounts of oxygen to maintain the standard 30\% dissolved oxygen concentration required for the expression of most recombinant proteins, the restricted dissolved amount of oxygen and inproast transcription by 5 -fold and produces 27-fold higher amount of rARU.

This research suggests that the restricted dissolved oxygen strategy combined with genetic engineering targeted at transcriptional repressors and ankyrin-like proteins could pave the way for the scaled-up production of. diftoxin A, which was shown to elicit protective immunity against as a possible vaccine agant pathosin. It is aline against this expression of recombinant to the from anaerobic origin or those with cell wall binding profiles. 

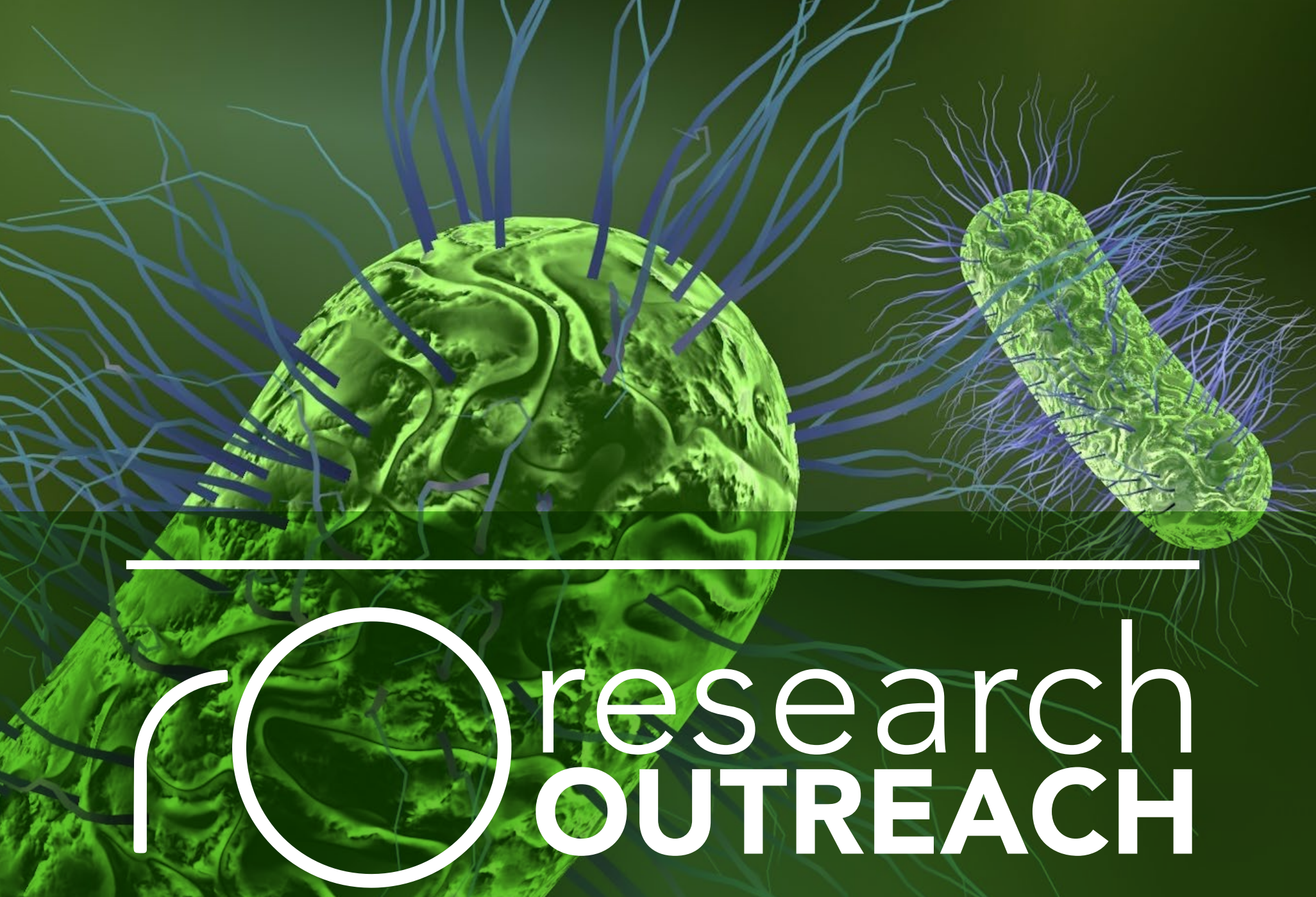

The public outreach magazine for the research community 20 The pulfic outreach

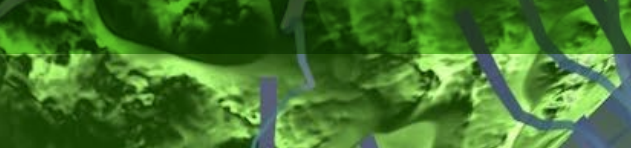

4. 3 researchoutreach.org
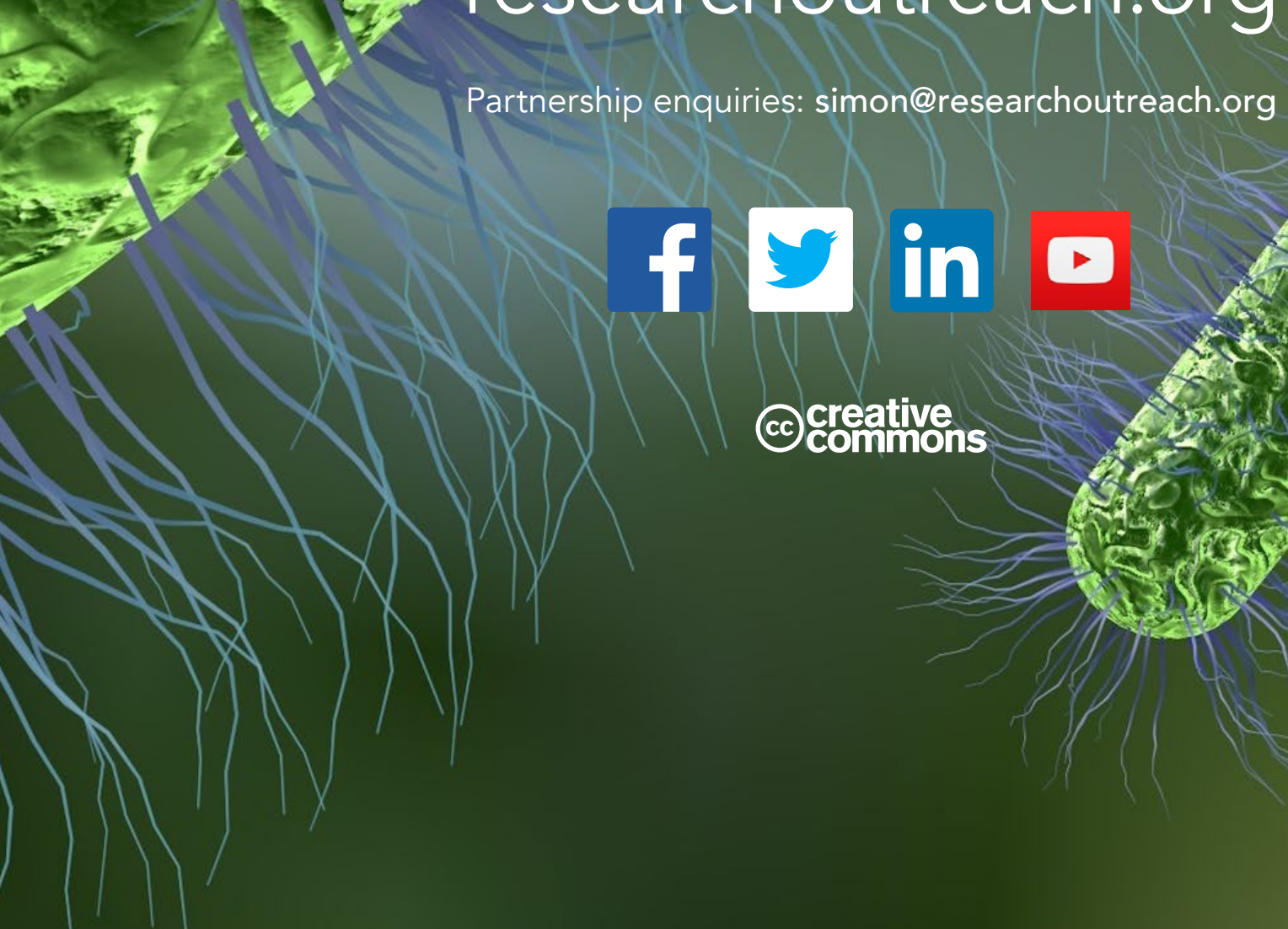

\section{$f y$ in 0}

c) creative 